\title{
Fungal Infections in the Era of Biologic Therapies
}

\author{
Nathan D. Gundacker ${ }^{1}$ John W. Baddley ${ }^{1,2}$
}

Published online: 27 March 2015

(C) Springer International Publishing AG 2015

\begin{abstract}
The number of patients receiving immunosuppressive agents has increased over the past decade and is expected to increase substantially in the near future. Biologic therapeutic antibodies, also referred to as "biologics," have become an important class of immunosuppressive drugs and are used widely in the fields of inflammation, cancer, autoimmunity, cardiovascular diseases, organ transplantation, infection, and ophthalmology. These drugs have had a substantial impact in the treatment of many diseases; however, there has also been a concern of increased risk of infection caused by bacterial, mycobacterial, fungal, and viral pathogens. This review will summarize available data on epidemiology of invasive fungal infections in patients receiving select biologic therapies.
\end{abstract}

Keywords Fungal infection $\cdot$ Biologic $\cdot$ TNF- $\alpha$ inhibitor . Rituximab $\cdot$ Pneumocystis $\cdot$ Histoplasmosis

This article is part of the Topical Collection on Mycology

John W. Baddley

jbaddley@uab.edu

Nathan D. Gundacker

ngundacker@uabmc.edu

1 Department of Medicine, Division of Infectious Diseases, University of Alabama at Birmingham, 1900 University Boulevard, 229 Tinsley Harrison Tower, Birmingham, AL 35294-0006, USA

2 Birmingham Veterans Affairs Medical Center, 700 South 19th Street, Birmingham, AL 35233, USA

\section{Introduction}

The number of patients receiving immunosuppressive agents has increased over the past decade and is expected to increase substantially in the near future. Biologic therapeutic antibodies have become an important class of drugs, with annual sales that exceeded \$27 billion in 2007 [1]. Key areas of biologic use and investigation include inflammation, cancer, autoimmunity, cardiovascular, organ transplantation, infection, and ophthalmology [2]. Although biologic drugs have had a substantial impact in the treatment of inflammatory and rheumatologic disorders and malignancies, there has also been an important safety concern: the use of biologics is associated with an increased incidence of serious infections caused by a broad spectrum of pathogens [3-8]. This review will summarize available data on epidemiology of invasive fungal infections in patients receiving select biologic therapies.

\section{Tumor Necrosis Factor- $\alpha$ (TNF- $\alpha$ ) Inhibitors (TNFI)}

TNF- $\alpha$, described in 1975 , is a cytokine that induces responses in both innate and adaptive immunity. It is expressed in $\mathrm{T}$ cells, macrophages, monocytes, and natural killer (NK) cells. TNF- $\alpha$ is synthesized initially as a 233 amino acid transmembrane precursor protein and later cleaved by TNF- $\alpha$ converting enzymes to the 157 amino acid residue soluble TNF- $\alpha$ that is released into circulation and mediates its biological activities [9]. The biologic activity of TNF- $\alpha$ requires the aggregation of three TNF-monomers to form trimeric TNF- $\alpha$, which then binds to two types of receptors: TNF-R1 (TNFRSF1A, CD120a) or TNF-R2 (TNFRSF1B, CD120b) which are expressed on most nucleated cells [9-11]. Soluble and transmembrane TNF- $\alpha$ bind to both receptors, but the biological activities of transmembrane TNF- $\alpha$ are mediated 
primarily through TNF-R2 [9]. The receptors exert multiple effects on the immune system, including (1) stimulation of inflammatory cytokines (IFN-g, IL-1, IL-6, IL-8); (2) upregulation of the expression of endothelial adhesion molecules (ICAM-1, VCAM-1, E-selectin), and chemokines (MIP-1 $\alpha$, RANTES); (3) effective granuloma maintenance; and (4) and mediation of cytotoxic activity $[9,11-13]$.

TNF- $\alpha$ inhibitors are important advances in the treatment of a number of inflammatory conditions, including rheumatoid arthritis (RA), seronegative spondyloarthropathies, and inflammatory bowel disease [14]. Five TNFI, infliximab, etanercept, adalimumab, certolizumab pegol, and golimumab are FDA approved for use in the USA (Table 1). The agents differ in structure, pharmacokinetic properties, tissue distribution, and functional properties.

\section{TNFI and Invasive Fungal Infection}

Despite the clinical benefits associated with TNFI, their use has been associated with an increased incidence of serious infections caused by a broad spectrum of pathogens $[4,7,8$, $15,16,17 \bullet$. Fortunately, fungal infections complicating TNFI use are relatively uncommon. Recently, there have been increasing reports of the association of TNFI and invasive fungal infection $[5,7,12,13,17 \bullet, 18,19,20 \bullet \cdot]$. A prospective study, the French RATIO registry, identified non-tuberculous opportunistic infections associated with TNF- $\alpha$ therapy [7]. Of 45 cases identified, 10 (22\%) were caused by fungal organisms.

Another large cohort study, Safety Assessment of Biologic ThERapy (SABER) Study, evaluated new users of TNFI and investigated the incidence of non-viral opportunistic infections among patients with RA, inflammatory bowel disease (IBD) and psoriasis, psoriatic arthritis, and ankylosing spondylitis [20••]. Overall, among 33,324 new users of TNFI, 80 non-viral opportunistic infections (OIs) were identified. Of these OIs, $32(40 \%)$ were caused by fungi, with an approximate crude incidence rate of 112 cases per 100,000 person-years. The most common fungal infections were pneumocystosis (16 cases) and histoplasmosis (9 cases) $[20 \bullet \cdot$.

A recent meta-analysis reported the risks of opportunistic infections from clinical trial data of biologic use in RA patients [17॰]. The authors found that biologic use did not significantly increase the risk for invasive fungal infections (odds ratio $2.58,95 \%$ confidence interval $0.68-11.91$ ) or Pneumocystis jiroveci pneumonia (odds ratio $1.77,95 \%$ confidence interval $0.42-7.47$ ) [17•]. A summary of specific fungal pathogens associated with TNFI follows.

\section{Aspergillosis}

TNF- $\alpha$ is important in host defense against Aspergillus fumigatus infections. Increased levels of TNF- $\alpha$ are found in mice infected with $A$. fumigatus conidia, and TNF- $\alpha$ enhances leukocyte killing of $A$. fumigatus [19, 21]. TNF/lymphotoxin double knockout mice are more susceptible to pulmonary invasive aspergillosis (IA), whereas mice exposed to TNF blockers are more susceptible to aspergillosis, resulting in increased fungal burden and mortality [10]. A recent report describes the importance of TNF-R1 polymorphisms and risk of IA [22].

Table 1 Selected biologic agents

\begin{tabular}{llll}
\hline Generic name & Trade name & Mechanism/type & Disease approvals \\
\hline $\begin{array}{l}\text { TNF- } \alpha \text { inhibitors } \\
\text { Infliximab }\end{array}$ & Remicade & Chimeric anti-TNF monoclonal antibody & RA, PsA, AS, IBD \\
Etanercept & Enbrel & Soluble TNF- $\alpha$ receptor fusion protein & RA, PsA, AS, juvenile RA, plaque \\
& & & psoriasis; not effective in IBD \\
Adalimumab & Humira & Human anti-TNF monoclonal antibody & RA, PsA, AS, IBD \\
Certolizumab pegol & Cimzia & PEGylated Fab' fragment of a humanized & Crohn's, RA \\
$\begin{array}{l}\text { Golimumab } \\
\text { Additional biologic agents }\end{array}$ & monoclonal antibody & RA, PsA, AS \\
Rituximab & Rimponi & Human anti-TNF monoclonal antibody & RA, CLL, non-Hodgkin's lymphoma, \\
Abatacept & & Monoclonal antibody against CD20 & Wegener's, microscopic polyangitis \\
Tofacitinib & Orencia & CTLA-4 ligand; T cell costimulation moderator & RA \\
Ustekinumab & Xeljanz & Janus kinase inhibitor & RA \\
Alemtuzumab & Stelara & Monoclonal antibody against IL-12 and IL-23 & Psoriasis \\
\hline
\end{tabular}

$T N F$ tumor necrosis factor, $R A$ rheumatoid arthritis, $P_{S A}$ psoriatic arthritis, $I B D$ inflammatory bowel disease, $C L L$ chronic lymphocytic leukemia 
The estimated incidence of aspergillosis is approximately 7 cases/100,000 persons treated [8, 18]. To date, greater than 75 cases of aspergillosis have been reported in patients receiving TNFI, mostly with use of infliximab [12, 23-25]. A summary of cases was published by Tsiodras and colleagues, who reviewed publications from January 1966 through June 1, 2007 to determine the association of fungal infections with TNF- $\alpha$ blockade [12]. They identified 64 cases of aspergillosis, mostly invasive pulmonary disease. The most common TNFI used was infliximab in 48 cases $(75 \%)$, followed by etanercept in $14(22 \%)$ and adalimumab in 3 cases $(3 \%)$. Of 24 cases reviewed with data available, 18 (75\%) had received TNFI for graft-versus-host disease (GVHD); 4 (17\%) for RA and $2(8 \%)$ for inflammatory bowel disease. The median number of infusions to diagnosis was 3 (interquartile range 2-6). Overall mortality was $82 \%$ among patients with GVHD; 3 of 4 cases with RA survived. Three additional cases were described as part of the prospective French RATIO registry, which identified 45 non-tuberculous opportunistic infections associated with TNF therapy [7]. Two cases were due to A. fumigatus, and sites of infection included sinus, colon, and lung.

\section{Coccidioidomycosis}

Tumor necrosis factor- $\alpha$ plays an important role in the control of infection by Coccidioides immitis. TNF- $\alpha$ is produced when human or rat cells are exposed to $C$. immitis spherules or arthroconidia and incubation of human monocytes with recombinant TNF- $\alpha$ results in increased fungicidal activity [26-28]. Thus, TNF- $\alpha$ inhibition may be expected to result in the increased incidence of coccidioidomycosis.

The incidence of coccidioidomycosis in patients receiving TNFI is estimated to be 5.58 per 100,000 persons treated with infliximab and 0.88 per 100,000 persons treated with etanercept [8, 18]. An early series (May 1998 through February 2003) by Bergstrom and colleagues described 13 cases among patients receiving TNFI from areas endemic for coccidioidomycosis [3]. One case received etanercept and the others received infliximab. The interval between TNFI and infection ranged from 1 to 96 weeks (mean 27 weeks). All patients had pneumonia on presentation, with $4(30.7 \%)$ having disseminated disease. Two patients had previous coccidioidomycosis and were thought to have reactivation disease. Five patients were hospitalized and 2 (15.4\%) of 13 died. The authors, by reviewing a cohort of inflammatory arthritis at one center, determined the incidence of coccidioidomycosis in patients receiving TNFI to be $1 \%$. Moreover, the risk of infliximab in development of symptomatic coccidioidomycosis when compared to other agents was greater (RR 5.23, $95 \%$ CI 1.54-17.71; $p<0.01)$. In a logistic regression model, use of infliximab was associated with development of symptomatic coccidioidomycosis after adjusting for methotrexate use, prednisone use, and age [3].

Taroumian and colleagues identified 44 patients with rheumatologic disease being treated with non-biologic diseasemodifying anti-rheumatic drugs (DMARDs) and/or biologic therapies at two medical centers in Tucson, AZ [29]. Of 36 patients receiving biologics at time of diagnosis of coccidioidomycosis, 21 were receiving infliximab, 8 adalimumab, 6 etanercept, and 1 abatacept. Twenty-nine patients had pulmonary coccidioidomycosis, 9 patients had disseminated disease, and 6 had asymptomatic coccidioidomycosis based on positive serology. With continuation or resuming biologic therapy after treatment, no patients had subsequent dissemination or complications of coccidioidomycosis.

\section{Histoplasmosis}

TNF- $\alpha$ is an important part of the protective immune response to both primary and secondary infection with Histoplasma capsulatum. In animal models, TNF- $\alpha$ is released after a pulmonary challenge with $H$. capsulatum cells, and TNF- $\alpha$ neutralized mice cannot control infection [30-32].

Histoplasmosis is one of the most common fungal infections in patients receiving TNFI $[5,18,20 \bullet \bullet$. The incidence of histoplasmosis in patients receiving TNFI has been estimated by Wallis and others with use of data from the FDA Adverse Event Reporting System (AERS) [8]. Wallis and colleagues collected data from cases reported to AERS from January 1998 through September 2002 and identified 40 cases of histoplasmosis. The estimated rate of histoplasmosis per 100,000 patients treated was 18.78 in patients treated with infliximab and 2.65 in patients treated with etanercept [8].

Soon after the release of the first TNFI in clinical practice, several case reports or series were published [33-35]. In 2008, the FDA reviewed 240 cases of histoplasmosis in patients treated with infliximab (207 cases), adalimumab (16 cases), or etanercept (17 cases). Most cases arose from endemic areas, and delay of diagnosis was not uncommon, occurring in at least 21 patients. Of those 21 patients, 12 (57\%) died.

Several large case series have been reported recently [5, 36]. Hage and colleagues described 19 patients diagnosed with histoplasmosis while receiving TNF- $\alpha$ inhibitors from 2000 to 2009 . Seventeen (89 \%) patients presented with progressive disseminated histoplasmosis; pulmonary involvement was present in $15(79 \%)$ patients [5]. Thirteen cases occurred in patients on infliximab, 3 on etanercept, and in 3 receiving adalimumab. Duration of treatment with TNF- $\alpha$ inhibitors ranged from $<1$ month to 3 years. Fifteen $(79 \%)$ patients required hospitalization, and all 19 patients recovered from infection. It is important to note that after discontinuing TNFI, clinical deterioration was observed in $8(42 \%)$ patients, 
perhaps related to immune reconstitution inflammatory syndrome (IRIS).

Olson and colleagues described 26 patients with RA who developed disseminated histoplasmosis from 1998 to 2009 at the Mayo Clinic in Rochester [36]. Of these, 15 were on TNFI, and median time to histoplasmosis was 15 months (range 2-132 months). Most patients were treated with at least 6 months of antifungal therapy. TNFIs were discontinued at the time of infection in 14 patients. TNFI therapy was restarted successfully in 4/15 with recurrence of disease in only 1 patient. Two deaths occurred in the case series, neither attributed to disseminated histoplasmosis.

\section{Pneumocystosis}

Initial clinical trials that led to the approval of TNFI did not identify cases of $P$. jirovecii pneumonia (PJP), but postmarketing surveillance identified several cases [37]. In 2007, Kaur and Mahl described 84 cases of PJP in patients on infliximab that had been reported to the FDA Adverse Event Reporting System from January 1998 through December 2003 [38]. This was notable for $27 \%$ mortality among the cases. Most patients had rheumatoid arthritis and developed pneumonia after an average of two infusions.

Numerous observational studies have reported the incidence of PJP among patients on TNFI [7, 20••, 39-43]. The incidence of PJP is usually less than 1 case per 1000 personyears of follow-up. In the French RATIO study, opportunistic infections (OIs) in patients on TNFI were gathered from reports to the regional pharmacovigilance centers, reports directly to pharmaceutical companies, and reports from physicians prescribing TNFI [7]. The study included patients on TNFI for inflammatory bowel disease, RA, psoriasis, psoriatic arthritis, or ankylosing spondylitis. Patients with histopathologic detection of $P$. jirovecii in a respiratory sample with compatible clinical disease were counted as a case. Only 5 cases of PJP were reported in the study from 57,711 patient-years of therapy from 2004 to 2006.

In the US SABER cohort, OIs occurring in patients on TNFI were identified retrospectively from inpatient and outpatient diagnosis codes submitted to four health insurance groups $[20 \bullet$ ]. The study included patients on TNFI for inflammatory bowel disease, RA, psoriasis, psoriatic arthritis, or ankylosing spondylitis. PJP was the most common fungal infection, with 16 cases identified in 28,493 person-years of exposure to TNFI. In the overall cohort, opportunistic infections were higher in new users of TNFI when compared to standard non-biologic therapies.

Several Japanese studies describing the incidence of PJP in patients on TNFI have reported significantly higher rates than what was seen in other studies $[42,43]$. Takeuchi and colleagues examined the incidence of adverse events in all
Japanese patients with RA for their first 6 months on infliximab as post-marketing surveillance [43]. The diagnosis of suspected PJP was made in $22(0.4 \%)$ patients. Twenty-one cases were diagnosed by a positive PCR for $P$. jirovecii DNA from BAL fluid, classified as suspected disease only. One case was diagnosed based on clinical findings alone. Of note, PJP incidence was greater than TB incidence $(0.4 \%)$.

The results from these Japanese studies have prompted further investigation into the incidence of PJP in rheumatoid arthritis patients in the USA [44]. The NIH conducted a population-based study to determine if the incidence of PJP in patients with RA had changed significantly from 1996 to 2007 using data from the Nationwide Inpatient Sample and the California Office of Statewide Health Planning and Development. They found no significant change in the number of patients with RA and PJP diagnoses over this time period. This study relied upon diagnostic codes reported to insurers for the identification of PJP cases which may underestimate the incidence of disease [44].

\section{Rituximab}

Rituximab is an anti-CD20 monoclonal antibody licensed for the treatment of RA, chronic lymphocytic leukemia (CLL), non-Hodgkin's lymphoma, Wegener's granulomatosis, and microscopic polyangiitis (Table 1) [45]. Rituximab binds the CD20 antigen on B lymphocytes and causes a reduction of B cells. The exact mechanism of action remains unclear, but direct signaling, complement-dependent cellular cytotoxicity, and antibody-dependent cellular cytotoxicity all appear to play a role in its efficacy [46].

There are a small number of reports regarding invasive fungal infections and use of rituximab. Vigna-Perez and colleagues evaluated 22 patients with SLE who received rituximab [47]. One patient died from severe metabolic acidosis related to diabetes as well as diffuse pulmonary histoplasmosis and mucormycosis in a coronary artery [47]. Several cases of cryptococcosis have been reported in the setting of rituximab use, corticosteroids, and other chemotherapeutic agents [48-50]. There have been several case reports of cryptococcal meningitis, which occurred 3-4 months after receiving rituximab [49-51].

A retrospective study of 30 patients who developed PJP after receiving rituximab was performed from 1998 to 2011 [52]. Hematologic malignancy was an underlying disease in $90 \%$ of patients; corticosteroids were used in $73 \%$ of chemotherapeutic regimens. Only $10 \%$ of patients developed PJP in the setting of single-agent rituximab. Hypoxemic respiratory failure was high, with $53 \%$ requiring ICU admission. The overall mortality of the cases was $30 \%$.

A US Veteran cohort of patients with RA (1998-2012) was investigated for OIs [53]. Patients received TNFIs, abatacept, 
or rituximab and all had previous TNFI use. Fungal infections were identified by diagnostic coding and laboratory results. Among 3777 patients contributing 5538 person-years, the incidence of fungal infection was 144 per 100,000 person-years. There were 8 fungal infections; 5 (3 PJP, 2 histoplasmosis) in patients who were receiving rituximab.

\section{Alemtuzumab}

Alemtuzumab is a humanized monoclonal antibody specific for CD52, which is present on lymphocytes, monocytes, and natural killer cells [54]. It produces a profound and prolonged depletion of lymphocytes, sometimes lasting up to 18 months [55]. Alemtuzumab is approved in the USA for the treatment of CLL and relapsing multiple sclerosis, but off-label uses also include treatment of autoimmune diseases and transplantation immunosuppression [56-59].
There are several cohorts and case series that have investigated fungal infections in patients who received alemtuzumab; however, it is difficult to attribute the risk of infection due to alemtuzumab, as many patients received other immunosuppressives [60-62]. Nath and colleagues followed 121 pancreas transplant recipients between February 2003 and February 2004 who received alemtuzumab for induction immunosuppression or who were converted from other therapies [60]. Eight patients $(6.6 \%)$ developed invasive fungal infection. The mean time to infection from start of alemtuzumab was 115.9 days (range 5-318 days). Organisms identified were Candida, Histoplasma, Aspergillus, and Cryptococcus. Five of eight patients were treated successfully; three patients died during treatment of the infection.

Peleg and colleagues analyzed 547 solid organ transplant recipients who received alemtuzumab from 2002 to 2004 to determine risk of opportunistic infections [61]. Those patients who received alemtuzumab for induction therapy had fewer

Table 2 Investigational biologic therapies

\begin{tabular}{|c|c|c|c|c|c|}
\hline Company & Generic name & Molecular format & Target & FDA approval phase & Proposed Indications \\
\hline Takeda & Vedolizumab & Human IgG1 & $\alpha 4 \beta 7$ integrin & Marketing approval & $\begin{array}{l}\text { Ulcerative colitis, Crohn's } \\
\text { disease }\end{array}$ \\
\hline Janssen & Siltuximab & Chimeric IgG1 & IL-6 & Marketing approval & $\begin{array}{l}\text { Multicentric Castleman } \\
\text { disease }\end{array}$ \\
\hline Lilly & Ramucirumab & Human IgG1 & VEGFR2 & Marketing approval & Gastric cancer \\
\hline Merck & Pembrolizumab & $\begin{array}{l}\text { Whole humanized } \\
\text { antibody }\end{array}$ & PD-1 & Marketing approval & Metastatic melanoma \\
\hline Bristol-Meyers Squib & Nivolumab & Human IgG4 & PD-1 & Marketing approval & Metastatic melanoma \\
\hline GSK & Mepolizumab & Humanized IgG1 & IL-5 & Marketing authorization & $\begin{array}{l}\text { Asthma, hypereosinophilic } \\
\text { syndrome, COPD with } \\
\text { eosinophilic bronchitis }\end{array}$ \\
\hline Eli Lilly and Co & Ixekizumab & Humanized IgG4 & IL-17a & $\begin{array}{l}\text { Regulatory submission } \\
\text { in } 2015\end{array}$ & $\begin{array}{l}\text { Rheumatoid arthritis, psoriatic } \\
\text { arthritis, psoriasis }\end{array}$ \\
\hline Novartis & Secukinumab & Human IgG1 & IL-17a & $\begin{array}{l}\text { Regulatory filing } \\
\text { in } 2015\end{array}$ & $\begin{array}{l}\text { Rheumatoid arthritis, psoriatic } \\
\text { arthritis, ankylosing } \\
\text { spondylitis, psoriasis }\end{array}$ \\
\hline Merck & Actoxumab+bezlotoxumab & Human IgG1 & $\begin{array}{l}\text { C. difficile } \\
\text { enterotoxin } \\
\text { A and B }\end{array}$ & Phase 3 & C. difficile infection \\
\hline Teva & Reslizumab & Humanized IgG4 & IL-5 & Phase 3 & Asthma/eosinophilic asthma \\
\hline AstraZeneca & Benralizumab & Humanized IgG1 & IL-5R & Phase 3 & Asthma \\
\hline Janssen & Sirukumab & Human IgG1 & IL-6 & Phase 3 & Rheumatoid arthritis \\
\hline Regeneron, Sanofi & Sarilumab & Human IgG1 & IL-6R subunit $\alpha$ & Phase 3 & Rheumatoid arthritis \\
\hline $\begin{array}{c}\text { Amgen, Kyowa } \\
\text { Hakko Kirin }\end{array}$ & Brodalumab & Human IgG2 & IL-17R & Phase 3 & $\begin{array}{l}\text { Plaque psoriasis, pustular } \\
\text { psoriasis, and psoriatic } \\
\text { erythroderma }\end{array}$ \\
\hline Merck & Tildrakizumab & Humanized IgG1 & IL-23 p19 subunit & Phase 3 & Plaque psoriasis \\
\hline Genentech & Ocrelizumab & Humanized IgG1 & CD20 & Phase 3 & Multiple sclerosis \\
\hline UCB & Epratuzumab & Humanized IgG1 & CD22 & Phase 3 & SLE \\
\hline Novartis & Bimagrumab & Human IgG1 & $\begin{array}{l}\text { Activin A receptor } \\
\text { type IIB }\end{array}$ & Phase $2 / 3$ & $\begin{array}{l}\text { Sporadic inclusion body } \\
\text { myositis }\end{array}$ \\
\hline
\end{tabular}

$P D-1$ programmed cell death receptor 1, IL-6 interleukin receptor 6, GSK GlaxoSmithKline, SLE systemic lupus erythematosus 
opportunistic infections than those who received it for rejection. Eighteen patients $(3.2 \%)$ were diagnosed with invasive fungal infection; 12 developing esophageal candidiasis. Two patients developed cryptococcosis, one with pulmonary disease, and one with meningitis. Two patients had invasive fungal sinusitis, one each with Aspergillus and Mucor species. Two intestinal transplant patients developed disseminated scedosporiosis and died as a result of the infection. There were no cases of Pneumocystis pneumonia.

Safdar and colleagues evaluated infections in solid organ transplant patients receiving alemtuzumab and, importantly, compared infections on the basis of induction immunosuppression regimen received [62]. Of 1026 patients in the cohort, 215 received basiliximab, 726 received alemtuzumab, and 85 anti-thymocyte or anti-lymphocyte globulin. Overall infection rates were similar in the three groups. Seventy-two (10\%) patients who received alemtuzumab induction developed a fungal infection, but the risk was not statistically different from the comparator groups. Greater than $75 \%$ of infections were caused by Candida species; 12 infections were caused by Aspergillus. Disseminated fungal infection was more common in the alemtuzumab group when compared to the basiliximab group (odds ratio 4.76; $95 \%$ confidence interval 1.58-14.28).

\section{Emerging and Investigational Biologic Therapies}

There are numerous biologic therapies under all phases of investigation, some of which target novel areas of the immune system (Tables 1 and 2) [63-66, 67•, 68, 69••, 70•]. Important pathways under investigation include cytokine signaling via the Janus kinase (JAK) and STAT pathways and the interleukin-17 immune axis $[64,65,68,70 \bullet]$.

The JAK-signal transducer and activator of transcription (STAT) pathway is a signal transduction pathway that is used by numerous cytokines $[67 \bullet, 68]$. Mutations or polymorphisms of JAKs have been associated with primary immunodeficiencies; myeloproliferative and hematologic malignancies; polycythemia; fungal, mycobacterial, and viral infections; and rheumatologic diseases [67•]. Ruxolitinib is a JAK1/JAK2 inhibitor approved for use in polycythemia vera. Tofacitinib inhibits JAK3 and JAK1 and is approved for RA. In preliminary trials with tofacitinib, there was increased frequency of local herpes zoster infections as well as pulmonary tuberculosis [67•]. Studies to assess the impact on fungal infections are ongoing.

IL-17a is implicated in immune-mediated inflammatory disorders such as psoriasis, psoriatic arthritis, rheumatoid arthritis, and ankylosing spondylitis $[63,64,66]$. Three monoclonal antibodies against the IL-17 pathway should be approved in the near future. Secukinumab and ixekizumab target the IL-17a ligand, while brodalumab targets the IL-17 receptor
$[63,66,71-73]$. IL-17's primary role in immunoprotection is against pathogens attacking mucous membranes including the mouth, lungs, and GI tract. Nasopharyngitis and urinary tract infections are adverse events reported in early clinical trials [66]. Fungal infections involving mucous membranes may propose risks for patients receiving these novel agents [71].

\section{Conclusions}

Biologic therapeutic antibodies have become an important class of drugs and will continue to be used widely in the treatment of inflammation, cancer, autoimmunity, cardiovascular diseases, organ transplantation, and ophthalmology. Fungal infections in patients who receive biologics are uncommon, but have potentially devastating consequences. Emerging drugs targeting novel facets of the immune system will bring new challenges to clinicians and warrant continued surveillance.

\section{Compliance with Ethics Guidelines}

Conflict of Interest Dr. Gundacker has no conflict of interest to declare.

Dr. Baddley is consulting for Pfizer, Astellas, Eli Lilly, and Merck. Dr. Baddley also has a research grant from BMS.

Human and Animal Rights and Informed Consent This article contains no studies with human or animal subjects performed by the author.

\section{References}

Papers of particular interest, published recently, have been highlighted as:

- Of importance

•- Of major importance

1. Scolnik PA. mAbs: a business perspective. mAbs. 2009;1(2): 179-84

2. Chan AC, Carter PJ. Therapeutic antibodies for autoimmunity and inflammation. Nat Rev. 2010;10(5):301-16.

3. Bergstrom L, Yocum DE, Ampel NM, et al. Increased risk of coccidioidomycosis in patients treated with tumor necrosis factor alpha antagonists. Arthritis Rheum. 2004;50(6):1959-66.

4. Curtis JR, Patkar N, Xie A, et al. Risk of serious bacterial infections among rheumatoid arthritis patients exposed to tumor necrosis factor alpha antagonists. Arthritis Rheum. 2007;56(4):1125-33.

5. Hage CA, Bowyer S, Tarvin SE, Helper D, Kleiman MB, Wheat LJ. Recognition, diagnosis, and treatment of histoplasmosis complicating tumor necrosis factor blocker therapy. Clin Infect Dis. 2012;50(1):85-92.

6. Patkar NM, Teng GG, Curtis JR, Saag KG. Association of infections and tuberculosis with antitumor necrosis factor alpha therapy. Curr Opin Rheumatol. 2008;20(3):320-6. 
7. Salmon-Ceron D, Tubach F, Lortholary O, et al. Drug-specific risk of non-tuberculosis opportunistic infections in patients receiving anti-TNF therapy reported to the 3-year prospective French RATIO registry. Ann Rheum Dis. 2011;12(4):219-27.

8. Wallis RS, Broder MS, Wong JY, Hanson ME, Beenhouwer DO. Granulomatous infectious diseases associated with tumor necrosis factor antagonists. Clin Infect Dis. 2004;38(9):1261-5.

9. Horiuchi T, Mitoma H, Harashima S, Tsukamoto H, Shimoda T. Transmembrane TNF-alpha: structure, function and interaction with anti-TNF agents. Rheumatology (Oxford, England). 2010;49(7):1215-28.

10. Rigby WF. Drug insight: different mechanisms of action of tumor necrosis factor antagonists-passive-aggressive behavior? Nat Clin Pract Rheumatol. 2007;3(4):227-33.

11. Ehlers S. Tumor necrosis factor and its blockade in granulomatous infections: differential modes of action of infliximab and etanercept? Clin Infect Dis. 2005;41 Suppl 3:S199-203.

12. Tsiodras S, Samonis G, Boumpas DT, Kontoyiannis DP. Fungal infections complicating tumor necrosis factor alpha blockade therapy. Mayo Clin Proc. 2008;83(2):181-94.

13. Smith JA, Kauffman CA. Endemic fungal infections in patients receiving tumour necrosis factor-alpha inhibitor therapy. Drugs. 2009;69(11):1403-15.

14. Smolen JS, Landewe R, Breedveld FC, et al. EULAR recommendations for the management of rheumatoid arthritis with synthetic and biological disease-modifying antirheumatic drugs. Ann Rheum Dis. 2010;69(6):964-75.

15. Crawford M, Curtis JR. Tumor necrosis factor inhibitors and infection complications. Curr Rheumatol Rep. 2008;10(5):383-9.

16. Mohan AK, Cote TR, Block JA, Manadan AM, Siegel JN, Braun MM. Tuberculosis following the use of etanercept, a tumor necrosis factor inhibitor. Clin Infect Dis. 2004;39(3):295-9.

17. Kourbeti IS, Ziakas PD, Mylonakis E. Biologic therapies in rheumatoid arthritis and the risk of opportunistic infections: a metaanalysis. Clin Infect Dis. 2014;58(12):1649-57. This paper is a large meta-analysis evaluating opportunistic infections from clinical trial data of patients with rheumatoid arthritis.

18. Wallis RS, Broder M, Wong J, Lee A, Hoq L. Reactivation of latent granulomatous infections by infliximab. Clin Infect Dis. 2005;41 Suppl 3:S194-8.

19. Filler SG, Yeaman MR, Sheppard DC. Tumor necrosis factor inhibition and invasive fungal infections. Clin Infect Dis. 2005;41 Suppl 3:S208-12.

20.• Baddley JW, Winthrop KL, Chen L, et al. Non-viral opportunistic infections in new users of tumour necrosis factor inhibitor therapy: results of the SAfety Assessment of Biologic ThERapy (SABER) Study. Ann Rheum Dis. 2014;73(11):1942-8. This article reviews opportunistic infections in a large cohort of patients receiving TNFI.

21. Mehrad B, Strieter RM, Standiford TJ. Role of TNF-alpha in pulmonary host defense in murine invasive aspergillosis. J Immunol. 1999;162(3):1633-40

22. Sainz J, Salas-Alvarado I, Lopez-Fernandez E, et al. TNFR1 mRNA expression level and TNFR1 gene polymorphisms are predictive markers for susceptibility to develop invasive pulmonary aspergillosis. Int J Immunopathol Pharmacol. 2010;23(2):423-36.

23. Salavert M, Bastida G, Peman J, Nos P. Opportunistic co-infection in a patient with Crohn's disease during infliximab (anti-TNFalpha) therapy. Rev Iberoam Micol. 2009;26(3):213-7.

24. Judson MA. Allergic bronchopulmonary aspergillosis after infliximab therapy for sarcoidosis: a potential mechanism related to T-helper cytokine balance. Chest. 2009;135(5):1358-9.

25. Leboime A, Berthelot JM, Allanore Y, et al. Sinus aspergilloma in rheumatoid arthritis before or during tumor necrosis factor-alpha antagonist therapy. Arthritis Res Ther. 2009;11(6):R164.
26. Slagle DC, Cox RA, Kuruganti U. Induction of tumor necrosis factor alpha by spherules of Coccidioides immitis. Infect Immun. 1989;57(7):1916-21.

27. Ampel NM. In vitro production of tumor necrosis factor-alpha by adherent human peripheral blood mononuclear cells incubated with killed coccidioidal arthroconidia and spherules. Cell Immunol. 1994;153(1):248-55.

28. Beaman L. Effects of recombinant gamma interferon and tumor necrosis factor on in vitro interactions of human mononuclear phagocytes with Coccidioides immitis. Infect Immun. 1991;59(11):4227-9.

29. Taroumian S, Knowles SL, Lisse JR, et al. Management of coccidioidomycosis in patients receiving biologic response modifiers or disease-modifying antirheumatic drugs. Arthritis Care Res. 2012;64(12):1903-9.

30. Deepe Jr GS. Modulation of infection with Histoplasma capsulatum by inhibition of tumor necrosis factor-alpha activity. Clin Infect Dis. 2005;41 Suppl 3:S204-7.

31. Allendoerfer R, Deepe Jr GS. Blockade of endogenous TNF-alpha exacerbates primary and secondary pulmonary histoplasmosis by differential mechanisms. J Immunol. 1998;160(12):6072-82.

32. Allendoerfer R, Deepe Jr GS. Regulation of infection with Histoplasma capsulatum by TNFR1 and -2 . J Immunol. 2000;165(5):2657-64.

33. Wood KL, Hage CA, Knox KS, et al. Histoplasmosis after treatment with anti-tumor necrosis factor-alpha therapy. Am J Respir Crit Care Med. 2003;167(9):1279-82.

34. Jain VV, Evans T, Peterson MW. Reactivation histoplasmosis after treatment with anti-tumor necrosis factor alpha in a patient from a nonendemic area. Respir Med. 2006;100(7):1291-3.

35. Lee JH, Slifman NR, Gershon SK, et al. Life-threatening histoplasmosis complicating immunotherapy with tumor necrosis factor alpha antagonists infliximab and etanercept. Arthritis Rheum. 2002;46(10):2565-70.

36. Olson TC, Bongartz T, Crowson CS, Roberts GD, Orenstein R, Matteson EL. Histoplasmosis infection in patients with rheumatoid arthritis, 1998-2009. BMC Infect Dis. 2011;11:145.

37. Bongartz T, Sutton AJ, Sweeting MJ, Buchan I, Matteson EL, Montori V. Anti-TNF antibody therapy in rheumatoid arthritis and the risk of serious infections and malignancies: systematic review and meta-analysis of rare harmful effects in randomized controlled trials. JAMA. 2006;295(19):2275-85.

38. Kaur N, Mahl TC. Pneumocystis jiroveci (carinii) pneumonia after infliximab therapy: a review of 84 cases. Dig Dis Sci. 2007;52(6): 1481-4.

39. Dixon WG, Watson K, Lunt M, Hyrich KL, Silman AJ, Symmons DP. Rates of serious infection, including site-specific and bacterial intracellular infection, in rheumatoid arthritis patients receiving anti-tumor necrosis factor therapy: results from the British Society for Rheumatology Biologics Register. Arthritis Rheum. 2006;54(8):2368-76.

40. Beukelman T, Xie F, Baddley JW, et al. Brief report: incidence of selected opportunistic infections among children with juvenile idiopathic arthritis. Arthritis Rheum. 2013;65(5):1384-9.

41. Greenberg JD, Reed G, Kremer JM, et al. Association of methotrexate and tumour necrosis factor antagonists with risk of infectious outcomes including opportunistic infections in the CORRONA registry. Ann Rheum Dis. 2010;69(2):380-6.

42. Komano Y, Harigai M, Koike R, et al. Pneumocystis jiroveci pneumonia in patients with rheumatoid arthritis treated with infliximab: a retrospective review and case-control study of 21 patients. Arthritis Rheum. 2009;61(3):305-12.

43. Takeuchi T, Tatsuki Y, Nogami Y, et al. Postmarketing surveillance of the safety profile of infliximab in 5000 Japanese patients with rheumatoid arthritis. Ann Rheum Dis. 2008;67(2):189-94. 
44. Louie GH, Wang Z, Ward MM. Trends in hospitalizations for Pneumocystis jiroveci pneumonia among patients with rheumatoid arthritis in the US: 1996-2007. Arthritis Rheum. 2010;62(12): 3826-7.

45. Maloney DG. Anti-CD20 antibody therapy for B-cell lymphomas. N Engl J Med. 2012;366(21):2008-16.

46. Weiner GJ. Rituximab: mechanism of action. Semin Hematol. 2010;47(2):115-23.

47. Vigna-Perez M, Hernandez-Castro B, Paredes-Saharopulos O, et al. Clinical and immunological effects of Rituximab in patients with lupus nephritis refractory to conventional therapy: a pilot study. Arthritis Res Ther. 2006;8(3):R83.

48. Ettahar N, Legout L, Ajana F, et al. Cryptococcal osteomyelitis in a patient with a lymphocytic leukemia treated with fludarabine-cyclophosphamide-rituximab. J Mycol Med. 2013;23(1):57-63.

49. Marchand T, Revest M, Tattevin P, et al. Early cryptococcal meningitis following treatment with rituximab, fludarabine and cyclophosphamide in a patient with chronic lymphocytic leukemia. Leuk Lymphoma. 2013;54(3):643-5.

50. Wingfield T, Jani M, Krutikov M, et al. Cryptococcal meningitis in an HIV-negative patient with rheumatoid arthritis treated with rituximab. Rheumatology (Oxford, England). 2011;50(9):1725-7.

51. Sanchez-Ojanguren J, Isern-Segura I, Chico-Chumillas C, Javaloyas-de-Morlius M. Cryptococcus neoformans meningoencephalitis in a patient treated with rituximab. Med Clin. 2009;133(4):157-8.

52. Martin-Garrido I, Carmona EM, Specks U, Limper AH. Pneumocystis pneumonia in patients treated with rituximab. Chest. 2013;144(1):258-65.

53. Baddley JW, Yang S, Brizendine K, Duvall S, et al. Rates of opportunistic infections among rheumatoid arthritis patients switching biologic therapy. Abstract 2093. 76th American College of Rheumatology Annual Meeting, November 9-14, 2012, Washington, DC.

54. Hale G, Xia MQ, Tighe HP, et al. The CAMPATH-1 antigen (CDw52). Tissue Antigens. 1990;35(3):118-27.

55. Hill-Cawthorne GA, Button T, Tuohy O, Jones JL, May K, Somerfield J, et al. Long term lymphocyte reconstitution after alemtuzumab treatment of multiple sclerosis. Neurol Neurosurg Psychiatry. 2012;83(3):298-304.

56. Kanda J, Lopez RD, Rizzieri DA. Alemtuzumab for the prevention and treatment of graft-versus-host disease. Int $\mathrm{J}$ Hematol. 2011;93(5):586-93.

57. Morgan RD, O'Callaghan JM, Knight SR, et al. Alemtuzumab induction therapy in kidney transplantation: a systematic review and meta-analysis. Transplantation. 2012;93(12):1179-88.

58. Havrdova E, Horakova D, Kovarova I. Alemtuzumab in the treatment of multiple sclerosis: key clinical trial results and considerations for use. Ther Adv Neurol Disord. 2015;8(1):31-45.
59. Reiff A. A review of Campath in autoimmune disease: biologic therapy in the gray zone between immunosuppression and immunoablation. Haemotology. 2005;10(2):79-93.

60. Nath DS, Kandaswamy R, Gruessner R, Sutherland DE, Dunn DL, Humar A. Fungal infections in transplant recipients receiving alemtuzumab. Transplant Proc. 2005;37(2):934.

61. Peleg AY, Husain S, Kwak EJ, Silveira FP, Ndirangu M, Tran J, et al. Opportunistic infections in 547 organ transplant recipients receiving alemtuzumab, a humanized monoclonal CD-52 antibody. Clin Infect Dis. 2007;44(2):204-12.

62. Safdar N, Smith J, Knasinski V, Sherkow C, Herrforth C, Knechtle $\mathrm{S}$, et al. Infections after the use of alemtuzumab in solid organ transplant recipients: a comparative study. Diagn Microbiol Infect Dis. 2010;66(1):7-15.

63. Yeremenko N, Paramarta JE, Baeten D. The interleukin-23/interleukin-17 immune axis as a promising new target in the treatment of spondyloarthritis. Curr Opin Rheumatol. 2014;26(4):361-70.

64. van den Berg WB, McInnes IB. Th17 cells and IL-17 a-focus on immunopathogenesis and immunotherapeutics. Semin Arthritis Rheum. 2013;43(2):158-70.

65. Reichert JM. Antibodies to watch in 2014. mAbs. 2014;6(1):5-14.

66. Genovese MC, Greenwald M, Cho CS, et al. A phase II randomized study of subcutaneous ixekizumab, an anti-interleukin-17 monoclonal antibody, in rheumatoid arthritis patients who were naive to biologic agents or had an inadequate response to tumor necrosis factor inhibitors. Arthritis Rheumatol. 2014;66(7):1693-704.

67. O'Shea JJ, Holland SM, Staudt LM. JAKs and STATs in immunity, immunodeficiency, and cancer. N Engl J Med. 2013;368(2):161-70. A review on biologic therapies targeting the JAK-STAT pathway.

68. O'Shea JJ, Kontzias A, Yamaoka K, Tanaka Y, Laurence A. Janus kinase inhibitors in autoimmune diseases. Ann Rheum Dis. 2013;72 Suppl 2:ii111-5.

$69 . \bullet$ Novosad SA, Winthrop KL. Beyond tumor necrosis factor inhibition: the expanding pipeline of biologic therapies for inflammatory diseases and their associated infectious sequelae. Clin Infect Dis. 2014;58(11):1587-98. A thorough review on the impact of biologic therapies and infection.

70. Reichert JM. Antibodies to watch in 2015. mAbs. 2015;7(1):1-8. A review of investigational biologic therapies.

71. Langley RG, Elewski BE, Lebwohl M, et al. Secukinumab in plaque psoriasis - results of two phase 3 trials. N Engl J Med. 2014;371(4):326-38.

72. Papp KA, Leonardi C, Menter A, et al. Brodalumab, an antiinterleukin-17-receptor antibody for psoriasis. N Engl J Med. 2012;366(13):1181-9.

73. Papp K, Leonardi C, Menter A, et al. Safety and efficacy of brodalumab for psoriasis after 120 weeks of treatment. J Am Acad Dermatol. 2014;71(6):1183-90. 6. 